\title{
Hearing loss in the developing world: Evaluating the iPhone mobile device as a screening tool
}

\author{
S Peer, MB BCh, FCORL (SA), MMed (Otol); J J Fagan, MB ChB, FCS (SA), MMed (Otol) \\ Division of Otorhinolaryngology, Faculty of Health Sciences, University of Cape Town, South Africa
}

Corresponding author: S Peer (shaziapeer@gmail.com)

\begin{abstract}
Background. Developing countries have the world's highest prevalence of hearing loss, and hearing screening programmes are scarce. Mobile devices such as smartphones have potential for audiometric testing.

Objectives. To evaluate the uHear app using an Apple iPhone as a possible hearing screening tool in the developing world, and to determine accuracy of certain hearing thresholds that could prove useful in early detection of hearing loss for high-risk populations in resource-poor communities.

Methods. This was a quasi-experimental study design. Participants recruited from the Otolaryngology Clinic, Groote Schuur Hospital, Cape Town, South Africa, completed a uHear test in three settings - waiting room (WR), quiet room (QR) and soundproof room (SR). Thresholds were compared with formal audiograms.

Results. Twenty-five patients were tested (50 ears). The uHear test detected moderate or worse hearing loss (pure-tone average (PTA) $>40 \mathrm{~dB}$ ) accurately with a sensitivity of $100 \%$ in all three environments. Specificity was $88 \%(\mathrm{SR}), 73 \%(\mathrm{QR})$ and $68 \%$ (WR). It was highly accurate in detecting high-frequency hearing loss $(2000,4000,6000 \mathrm{~Hz})$ in the QR and SR with 'good' and 'very good' kappa values, showing statistical significance $(p<0.05)$. It was moderately accurate in low-frequency hearing loss $(250,500,1000 \mathrm{~Hz})$ in the SR, and poor in the QR and WR.

Conclusion. Using the iPhone, uHear is a feasible screening test to rule out significant hearing loss (PTA $>40 \mathrm{~dB}$ ). It is highly sensitive for detecting threshold changes at high frequencies, making it reasonably well suited to detect presbycusis and ototoxic hearing loss from HIV, tuberculosis therapy and chemotherapy. Portability and ease of use make it appropriate to use in developing world communities that lack screening programmes.
\end{abstract}

S Afr Med J 2015;105(1):35-39. DOI:10.7196/SAMJ.8338

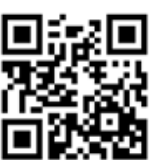

Hearing disability is one of the world's major health problems. ${ }^{[1]}$ An estimated 360 million people currently live with disabling hearing loss (defined as hearing loss $>40 \mathrm{~dB}$ in the better-hearing ear in adults $(>15$ years $)$ and $>30 \mathrm{~dB}$ in the better-hearing ear in children $(0-14$ years). ${ }^{[2]}$ The prevalence is greatest in the developing world, where the majority of deaf people reside. ${ }^{[1]}$ Hearing loss is more common in adults than in children, constituting $91 \%$ of all cases. ${ }^{[2]}$ The highest prevalence rates are found in sub-Saharan Africa (15.7\%) and South Asia (17\%). ${ }^{[1]}$ Failure to deal with this health challenge perpetuates economic and health decline, as confirmed by the inverse relationship between the prevalence of hearing loss and gross national income. ${ }^{[2]}$ For the individual, hearing loss leads to poor communication and social isolation. ${ }^{[3]}$ The deaf are less likely to obtain employment than people with normal hearing, and those who are employed often earn incomes in the lowest bracket. ${ }^{[1,3]}$

Screening programmes directed at high-risk groups are necessary. If effective, they can reduce morbidity from hearing loss through early detection and rehabilitation. ${ }^{[4]}$ Highly sensitive tools that yield lower false-positive and higher true-negative results are ideal for screening and monitoring hearing. There are currently few screening programmes for high-risk groups in the developing world. ${ }^{[5]}$ High-risk groups include the elderly and patients receiving potentially ototoxic medications: chemotherapeutic agents, ${ }^{[6]}$ second-line antituberculosis (TB) regimens and antiretrovirals (ARVs). ${ }^{[1,7,8]}$ These groups are at risk of developing high-frequency hearing loss, as explained below.

\section{High-risk groups Presbycusis}

Presbycusis (adult-onset hearing loss) is generally underdiagnosed and undertreated. This leads to late detection, disease progression and poor rehabilitation. ${ }^{[9,10]}$ Such hearing loss is predominantly high frequency and sensorineural. Lin et al. ${ }^{[11,12]}$ reported that older adults with hearing loss are more likely to develop cognitive impairment and dementia than their contemporaries without hearing loss. Regular monitoring and early detection and treatment of presbycusis would maintain an existing quality of life.

\section{HIV and TB therapy}

Currently approximately 25 million people in South Africa (SA) are HIV-positive. ${ }^{[13]}$ Hearing loss can be caused by HIV and/or its treatment; both are implicated in sensorineural hearing loss. ${ }^{[7]}$ Treatment aimed at opportunistic infections and antineoplastic therapy can have synergistic hearing effects on patients receiving ARVs. ${ }^{[7,8]}$ TB therapy is one such treatment, and TB is an increasing problem in sub-Saharan Africa owing to co-infection with HIV. There are increasing numbers of cases of multidrug-resistant (MDR) TB in $\mathrm{SA},{ }^{[8,14]}$ the treatment of which involves injectable aminoglycosides that are known to cause ototoxicity ${ }^{[8,15]}$ via a synergistic ototoxic effect together with ARVs on the outer hair cells of the cochlea, leading to high-frequency sensorineural hearing loss (HFSNHL). ${ }^{[15]}$

\section{Chemotherapy}

Cancer rates are increasing rapidly in the developing world. ${ }^{[16]}$ Cisplatin, a commonly used antineoplastic drug, is known to cause irreversible dose-dependent ototoxicity ${ }^{[6,17]}$ leading to HFSNHL. Noise exposure may result in a three-fold increased risk of hearing loss with cisplatin. ${ }^{[17,18]}$

Ototoxicity monitoring is aimed at preventing or minimising the progression of hearing loss through prospective hearing 
assessments. It is the most reliable method of detecting ototoxicity prior to development of symptomatic hearing $\operatorname{loss}^{[19]}$ and permits clinicians to counsel patients, possibly modify treatment regimens, and rehabilitate hearing. Three methods of testing can be utilised, namely conventional audiology, high-frequency audiometry (HFA) and otoacoustic emissions. HFA has significant change criteria and excellent specificity and sensitivity, ${ }^{[8,19]}$ but is generally not available in developing countries.

\section{Advances in screening techniques}

While screening programmes traditionally involve audiologists testing patients in soundproof booths, alternative screening methods have evolved in recent years. Telehealth projects allow patients in remote areas to be tested using high-speed internet. ${ }^{[20]}$ Portable audiometers are also becoming more advanced, and provide good-quality diagnostic audiometry in any environmental setting. ${ }^{[21]}$

Global mobile phone penetration of the world's population is at $85 \%,{ }^{[22]}$ and 1 in 5 Africans own smartphones. Automated hearing screening apps, using commercially available technology, present an opportunity to address the global problem of hearing loss. Yeung et al. ${ }^{[23]}$ from the Children's Hospital of Eastern Ontario reported on the first portable clinical 'conditioned play' iPad-based audiometer for the paediatric population. Their study shows promise in focusing on the shortcomings of existing play audiometry. Other authors have reported on the Apple uHear app using an iPodTouch device, with varying results. uHear is a hearing program devised by Unitron and Apple for use on any touchinterface Apple device, and is freely available for download from the iTunes Apple store.

Szudek et al. ${ }^{[24]}$ were the first of three groups to evaluate this app as a potential hearing screening tool. Using an iPodTouch, participants were tested in different noise level environments. Pure-tone averages (PTAs) calculated as a mean at thresholds of 500, 1000,2000 and $4000 \mathrm{~Hz}$ from the uHear application were compared with the formal audiogram. uHear was able to correctly diagnose the presence of moderate or worse hearing loss (PTA $>40 \mathrm{~dB}$ ) in 100 participants, with a sensitivity of $98 \%$ and a specificity of $82 \%$ in the clinic. In the soundproof room, sensitivity improved to $100 \%$ and specificity to $90 \%$. KhozaShangase et al. ${ }^{[25]}$ sampled a group of children using the uHear application on an iPodTouch in a school environment. PTAs (mean at thresholds of 500,1 000 and $2000 \mathrm{~Hz}$ ) from the uHear test were compared with PTAs from formal audiograms. They reported large deviations in the lower frequencies, standard deviations of $>10 \mathrm{~dB}$ at individual thresholds and a $34 \%$ increased pick-up rate of hearing loss. The third study, by Handzel et al., ${ }^{[26]}$ tested participants with unilateral sudden sensorineural hearing loss using the uHear on an iPodTouch, and reported a sensitivity of $76 \%$ and a specificity of $91 \%$ when compared with a formal audiogram. Inaccurately elevated thresholds at low frequencies using uHear were also detected, corroborating the findings of the previous study. ${ }^{[25]}$ Interestingly, uHear reflected hearing thresholds more accurately in mid- to high frequencies than in low ones, and deviations in low frequencies were less pronounced in the abnormal-hearing (diseased) ear. These two observations were also reported by Szudek et al. ${ }^{[24]}$ These studies have highlighted two important points. Firstly, it is possible to rule out moderate or disabling hearing loss. ${ }^{[24,26]}$ Secondly, even though low frequencies may be inaccurately elevated, ${ }^{[24-26]}$ the degree of hearing loss in the participant with abnormal hearing can possibly be predicted in the high frequencies. ${ }^{[24,26]}$

\section{Objectives}

To determine the accuracy of: (i) the iPhone using the uHear app as a screening tool for moderate or worse hearing loss (PTA $>40 \mathrm{~dB}$ ), where PTA is the mean at thresholds of 500,1 000, 2000 and $4000 \mathrm{~Hz}$; and (ii) individual thresholds at certain frequencies, especially the high frequencies (which may be of use in high-risk population groups)

\section{Methods}

The study was approved by the Research Ethics Committee, Faculty of Health Sciences, University of Cape Town, SA. The design was quasi-experimental. Study participants were consecutively recruited from patients attending the Otolaryngology Clinic at Groote Schuur Hospital, Cape Town. The inclusion criteria were age 15 - 80 years and having had a formal audiogram performed by a trained audiologist at Groote Schuur Hospital in the previous 2 weeks. Exclusion criteria included otorrhoea, visual impairment, learning disability, and poor gross motor skills rendering the patient incapable of tapping the screen. Non-English-speaking patients were not excluded if they understood the instructions after the task had been explained to them. All participants gave informed consent. The results obtained from the iPhone testing did not affect treatment plans.

\section{Test instruments}

An iPhone 4 mobile device (iOS 4.2) was used. The uHear app (version 1.0) was downloaded from iTunes on to the device at no cost. The application allows users to test their pure-tone airconduction hearing sensitivity as well as speech in noise. For this study, participants completed only the hearing sensitivity test, which employs a $267 \mathrm{~ms}$ pulse duration, with a ' $10 \mathrm{~dB}$ down and $5 \mathrm{~dB}$ up' approach. The time delay between tone presentations is randomised to prevent anticipation, and the lowest threshold with two positive responses of three excursions is recorded as the hearing sensitivity.

'Earbud' earphones that come standard with the device were used, and the buds were cleaned with an alcohol swab before and after every test in the presence of the participant. With reference to calibration, a single iPhone was used to test all participants. The same uHear application (version 1.0) was used for all participants tested. Ambient noise levels in three test environments were measured at regular intervals with the OMD G45 71-6229 Psio sound level meter, providing an adequate measure of quality control. Sound levels for the respective environment complied with the South African Bureau of Standards requirements for all three environments.

\section{Technique}

Otoscopic examination was performed to exclude otorrhoea and wax impaction. Infection control was maintained during testing. All participants had formal audiograms done, and were tested in three different settings with the iPhone, i.e. the waiting room (WR), a quiet room $(\mathrm{QR})$ and the soundproof room (SR).

\section{Instructions to participants}

Participants were given the device and the earphones to insert. The uHear app was selected, earphone connectivity confirmed and the participant advised to 'tap the screen when a sound is heard'. The programme plays a series of pure tones of varying levels so that a threshold can be determined. On hearing a sound, the participant was expected to follow the commands. The duration of the test is 6 minutes. No audiologist is normally required, as the program is a selfassessment one. However, as this was a formal study an investigator was present to ensure that the test was completed. 


\section{Data analysis}

For hearing screening, the presence or absence of moderate or worse hearing loss (PTA $>40 \mathrm{~dB}$ ) in each ear was determined by formal audiometry, as $40 \mathrm{~dB}$ is considered the critical hearing threshold for disabling hearing loss according to the World Health Organization, and warrants further investigation.

The iPhone uHear PTAs in all three settings were compared with the formal audiogram PTAs, where the PTA is calculated as a mean of thresholds (hearing in $\mathrm{dB}$ ) at 500, 1000,2000 and $4000 \mathrm{~Hz}$. These data were captured into $2 \times 2$ tables to calculate sensitivity, specificity and accuracy ratios.

Additionally, in view of the possibility for agreement by 'chance', kappa analysis was performed on iPhone thresholds from all three settings when compared with the formal audiogram to detect agreement at all six frequencies. Kappa values range from $<0.2$ to 1.0 depending on how well two thresholds correlate with each other at a particular frequency. The best correlation kappa value, i.e. 'very good', is one that is closest to 1. This describes how well the thresholds at a particular frequency correlated with the thresholds at the same frequency of the gold standard, the formal audiogram, and values this correlation according to the range described in Table 1.

Table 1. Kappa range of values and their correlation

\begin{tabular}{ll}
\hline Kappa values & 'Agreement'/comparison \\
\hline$<0.2$ & Poor \\
$0.21-0.4$ & Fair \\
$0.41-0.6$ & Moderate \\
$0.61-0.8$ & Good \\
$0.81-1.0$ & Very good
\end{tabular}

Table 2. Participants' demographics and hearing loss (as graded by the ASHA degree of severity of hearing loss)

\begin{tabular}{ll}
\hline Participants, $N$ & 25 \\
Age (years), mean (range) & 43 (15 - 86) \\
Age distribution (years), $n$ & \\
15 - 20 & 4 \\
21 - 40 & 7 \\
41 - 60 & 6 \\
61 - 80 & 8 \\
Gender, $n$ & 13 \\
Male & 12 \\
Female & \\
Ears with PTA (dB), $n$ & 24 \\
$\leq 25$ (normal) & 18 \\
26 - 40 (mild loss) & 5 \\
41 - 55 (moderate loss) & 2 \\
56 - 70 (moderate to severe loss) & 1 \\
71 - 90 (severe loss) & 0 \\
$\geq 91$ (profound loss) & \\
ASHA = American Speech-Language-Hearing Association; PTA = pure-tone average. &
\end{tabular}

\section{Results}

Thirty patients met the inclusion criteria and were recruited, of whom five were excluded for incomplete testing (unrelated to the device or software). Twenty-five patients were therefore fully tested (50 ears in total). Participants' demographics are described in Table 2.

Accuracy of the iPhone uHear as a screening test. All ears with moderate or worse hearing loss (PTA $>40 \mathrm{~dB}$ ) were detected in all three settings. This translated to a sensitivity of $100 \%$. Of the 42 ears without moderate or worse hearing loss (PTA $\leq 40 \mathrm{~dB}$ ), 15 had moderate or worse hearing in the WR (specificity 64\%), 11 had moderate or worse hearing in the QR (specificity 74\%), and 5 had moderate or worse hearing loss in the SR (specificity 88\%) (Table 3). Accuracy in the WR was calculated as 70\%, that in the QR as $78 \%$ and that in the SR as $90 \%$.

Accuracy of the iPhone uHear thresholds at all six frequencies. Kappa analysis compared the 'agreement' of the iPhone thresholds in all three rooms with the formal audiogram thresholds as reflected in Table 4. The iPhone was highly accurate at the high frequencies (2 000, 4000 and $6000 \mathrm{~Hz}$ ), where there was 'good' and 'very good' correlation in the SR and QR (Table 4). This was found to be statistically significant $(p<0.05)$. The iPhone did not correlate well at low frequencies in all three rooms, being 'fair to moderate' in the SR and 'poor to moderate' in the QR. The WR showed 'poor to fair' correlation at low frequencies and 'moderate' correlation at high frequencies.

The iPhone uHear therefore reflected thresholds more accurately in the mid- to high frequencies than in the low frequencies. Furthermore, the SR did not eliminate this low-frequency inaccuracy. These two salient findings correlate with all three studies reviewed. ${ }^{[24-26]}$

\section{Discussion}

Our study is one of the first to evaluate smartphone-assisted audiometry as a hearing screening tool for populations that currently have no access to formal audiometry. Results indicate that the iPhone uHear application is reasonably accurate at screening for moderate or worse (disabling) hearing loss. We found a sensitivity of $100 \%$, with a very high negative predictive value, implying an ideal test for screening. The highest test accuracy (90\%) was found in an SR, with a specificity of $88 \%$, rendering the least false positives. This highlights the need for caution when testing in a WR setting.

Table 3. Accuracy of the iPhone as a screening test in three different settings (WR, QR, SR), compared with the formal audiogram

\begin{tabular}{lll}
\hline & \multicolumn{2}{c}{ Formal audiogram, $\boldsymbol{n}$} \\
\cline { 2 - 3 } iPhone, $\boldsymbol{n}$ & PTA $\leq \mathbf{4 0} \mathbf{~ d B}$ & PTA $>\mathbf{4 0} \mathbf{~ d B}$ \\
\hline WR & 17 & 0 \\
PTA $\leq 40 \mathrm{~dB}$ & 25 & 8 \\
PTA $>40 \mathrm{~dB}$ & & 0 \\
QR & 31 & 8 \\
PTA $\leq 40 \mathrm{~dB}$ & 11 & 0 \\
PTA $>40 \mathrm{~dB}$ & & 8 \\
SR $\quad$ & 37 & 5 \\
PTA $\leq 40 \mathrm{~dB}$ & & \\
PTA $>40 \mathrm{~dB}$ & &
\end{tabular}


Table 4. Kappa values (range of values and their correlation are set out in Table 1) seen with iPhone and formal audiogram thresholds in three different settings (WR, QR, SR)

\begin{tabular}{|c|c|c|c|}
\hline & \multicolumn{3}{|c|}{ Formal audiogram, low frequencies $(\mathrm{Hz})$} \\
\hline $\begin{array}{l}\text { iPhone, } \\
\text { low frequencies }(\mathrm{Hz})\end{array}$ & 250 & 500 & 1000 \\
\hline \multicolumn{4}{|l|}{ WR } \\
\hline 250 & -0.0516 & 0.2217 & 0.4123 \\
\hline 500 & 0.0044 & 0.2399 & 0.4997 \\
\hline 1000 & -0.0693 & 0.2526 & 0.5126 \\
\hline \multicolumn{4}{|l|}{ QR } \\
\hline 250 & -0.0101 & 0.2751 & 0.5677 \\
\hline 500 & 0.0134 & 0.3102 & 0.5113 \\
\hline 1000 & -0.0239 & 0.212 & 0.5207 \\
\hline \multicolumn{4}{|l|}{ SR } \\
\hline 250 & 0.097 & 0.5309 & 0.4386 \\
\hline 500 & 0.1309 & 0.5283 & 0.5316 \\
\hline \multirow[t]{2}{*}{1000} & 0.0113 & 0.481 & $0.66(p=0.000)$ \\
\hline & \multicolumn{3}{|c|}{ Formal audiogram, high frequencies $(\mathrm{Hz})$} \\
\hline \multicolumn{4}{|l|}{ iPhone, } \\
\hline high frequencies $(\mathrm{Hz})$ & 2000 & 4000 & 6000 \\
\hline \multicolumn{4}{|l|}{ WR } \\
\hline 2000 & 0.496 & 0.663 & 0.4261 \\
\hline 4000 & 0.4667 & 0.339 & 0.5529 \\
\hline 6000 & 0.484 & 0.187 & 0.517 \\
\hline \multicolumn{4}{|l|}{ QR } \\
\hline 2000 & $0.73(p=0.001)$ & 0.5122 & 0.5535 \\
\hline 4000 & 0.4471 & $0.74(p=0.000)$ & $0.69(p=0.000)$ \\
\hline 6000 & 0.4339 & $0.69(p=0.0092)$ & $0.79(p=0.0026)$ \\
\hline \multicolumn{4}{|l|}{ SR } \\
\hline 2000 & $0.75(p=0.000)$ & $0.72(p=0.0039)$ & $0.69(p=0.005)$ \\
\hline 4000 & $0.73(p=0.000)$ & $0.85(p=0.000)$ & $0.89(p=0.000)$ \\
\hline 6000 & $0.77(p=0.007)$ & $0.92(p=0.0010)$ & $0.94(p=0.000)$ \\
\hline
\end{tabular}

The iPhone uHear application was found to be highly accurate for detecting high-frequency hearing loss in the QR and SR in patients with various levels of hearing. The iPhone uHear application could therefore be used to screen those at high risk of developing highfrequency hearing loss through early detection of abnormal or worsening thresholds.

The iPhone uHear application is therefore an appropriate tool to screen for disabling hearing loss, and to detect high-frequency hearing loss in the abovementioned highrisk groups in poorly resourced communities that have limited access to healthcare. In particular, it can be used to screen and monitor hearing in drug-related ototoxicity where high-frequency assessments of both conventional audiometry and HFA have a more accurate positive and negative predictive values, thereby rendering the testing more accurate. Environmental noise may have been a cause for poor results at the lower frequencies. Testing can possibly be improved by using background noise eliminators. Inset earphones are theoretically recommended as an effective way to reduce ambient noise. Being placed within the external ear canal, they can provide $30-40 \mathrm{~dB}$ attenuation of ambient noise. ${ }^{[27]}$ In our study, inaccuracy can be related to insertion depth; 'earbud' earphones sit just beyond the concha at the entrance to the external ear canal, which could explain the poor low-frequency outcomes. In future, testing that incorporates inset earphones cupped by circumaural ear covers with integrated ambient noise level monitoring that eliminates or adjusts testing to accommodate background noise could be done. Although this would be ideal, it does counter the intention to screen using a device with its standard hardware. A single iPhone device was used for this study; to avoid problems relating to inter-device reliability, every iPhone may have to be calibrated.

\section{Conclusions}

The sensitivity of the uHear app used with the iPhone is adequate to screen for disabling hearing loss, and has good accuracy to high-frequency hearing loss in SRs and QRs. Early detection of hearing loss with hearing screening programmes is therefore possible using mobile digital technology. A mobile, non-operator-dependent method used to screen for disabling hearing loss and detect early high-frequency threshold changes, such as an Apple iPhone, can help overcome the lack of trained audiologists or available infrastructure in developing countries. ${ }^{[5]}$ Because of the portability of the device, hearing screening with the iPhone uHear test is taken directly to the patient and is an opportune way to utilise existing community health and educational facilities. Oncology units, ARV rollout centres and TB hospitals with MDR/ extensively drug-resistant patients are ideal centres in which to use this technology. In addition, satellite clinics that regularly dispense chronic medication to older adults can also be considered.

Disclaimer. The Division of Otorhinolaryngology, Groote Schuur Hospital, and the authors do not have any affiliation to the Apple or Unitron companies. 


\section{References}

1. Stevens G, Flaxman S, Brunskill E, et al. Global and regional hearing impairment prevalence: An analysis of 42 studies in 29 countries. Eur J Public Health 2011;23(1):146-152. [http://dx.doi. org/10.1093/eurpub/ckr176]

2. World Health Organization. WHO Global Estimates on Prevalence of Hearing Loss. Geneva: WHO 2012. http://www.who.int/pbd/deafness/WHO_GE_HL.pdf?ua=1 (accessed 20 November 2014).

3. Tucci DL, Merson M, Wilson BS. A summary of the literature on global hearing impairment: Current status and priorities for action. Otol Neurotol 2009;31(1):31-41. [http://dx doi.org/10.1097/ MAO.0b013e3181c0eaec]

4. Swanepoel DW, Storbeck C, Friedland P. Early hearing detection and intervention in South Africa. International Journal of Pediatric Otorhinolaryngology 2009;73(6):783-786. [http://dx.doi. org/10.1016/ji.jporl.2009.01.007]

5. Fagan JJ, Jacobs M. Survey of ENT services in Africa: Need for a comprehensive intervention. Global Health Action 2009;2:1-7. [http://dx.doi.org/10.3402/gha.v2i0.1932]

6. Whitehorn $\mathrm{H}$, Sibanda M, Lacerda M, et al. High prevalence of cisplatin-induced ototoxicity in Cape Town, South Africa. S Afr Med J 2014;104(4):288-291. [http://dx.doi.org/10.7196/SAMJ.7389]

7. Katijah KS. Is there a need for ototoxicity monitoring for HIV:AIDs. Afr J Pharm Pharmacol 2010;4(9):574-579.

8. Harris T, Peer S, Fagan JJ. Audiological monitoring for ototoxic tuberculosis, human immunodeficiency virus and cancer therapies in a developing world setting. J Laryngol Otol 2012;126(6):548-551. [http:// dx.doi.org/10.1017/S0022215112000357]

9. Pacala JT, Yueh B. Hearing deficits in the older patient: 'I didn't notice anything. JAMA 2012;307(11):1185-1194. [http://dx.doi.org/10.1001/jama.2012.305]

10. Yueh B, Shapiro N, MacLean CH, Shekelle PG. Screening and management of adult hearing loss in primary care scientific review. JAMA 2003;289(15):1976-1985. [http://dx.doi.org/10.1001/ jama.289.15.1976]

11. Lin FR, Metter EJ, An Y, et al. Hearing loss and cognition in the Baltimore Longitudinal Study of Aging. Neuropsychology 2011;25(6):763-770. [http://dx.doi.org/10.1037/a0024238]

12. Lin FR, O'Brien RJ, Resnick SM, et al. Hearing loss and incident dementia. Arch Neurol 2011:68(2):214220. [http://dx.doi.org/10.1001/archneurol.2010.362]

13. World Health Organization. UN Core Epidemioloigical Slides. 2013. http://www.unaids.org/en/ media/unaids/contentassets/documents/epidemiology/2013/gr2013/201309_epi_core_en.pd (accessed 20 November 2014)

14. World Health Organization. Multidrug and Extensively Drug Resistant TB (M/XDR-TB): 2010 Report on Surveillance and Response. Geneva: WHO, 2010.

15. Harris T, Bardien S, Schaaf HS, et al. Aminoglycoside-induced hearing loss in HIV-positive and HIVnegative multidrug-resistant tuberculosis patients. S Afr Med J 2012;102(6):363-366.
16. Farmer P, Frenk J, Knaul FM, Shulman LN, et al. Expansion of cancer care and control in countries of low and middle income: A call to action. Lancet 2010;376(9747):1186-1193. [http://dx.doi. org $/ 10.1016 /$ S0140-6736(10)61152-X]

17. Bokemeyer C, Berger CC, Hartmann JT, et al. Analysis of risk factors for cisplatin-induced ototoxicity in patients with testicular cancer. Br J Cancer 1998;77(8):1355-1362. [http://dx.doi.org/10.1038/ bjc.1998.226

18. Fausti SA, Schaffer HJ, Olson DJ, et al. Audiometric monitoring for early detection of aminoglycoside ototoxicity. J Infect Dis 1992;165(6):1026-1032. [http://dx.doi.org/10.1093/infdis/165.6.1026]

19. American Academy of Audiology. Position Statement and Clinical Practice Guidelines: Ototoxicity Monitoring.October 2009. http://audiology-web.s3.amazonaws.com/migrated/OtoMonGuidelines.
. pdf_539974c40999c1.58842217.pdf (accessed 20 November 2014).

20. Swanepoel DW, Clark JL, Koekemoer D, et al. Telehealth in audiology: The need and potential to reach underserved communities. Int J Audiol 2010;49(3):195-202. [http://dx.doi. org/10.3109/1499202

21. Maclennan-Smith F, Swanepoel DW, Hall JW. Validity of diagnostic pure-tone audiometry without a sound-treated environment in older adults. Int J Audiol 2013;52(2):66-73. [http://dx.doi.org/10.3109 /14992027.2012.736692

22. Kujawski M. Finally some 2012 statistics for the African mobile phone market. 2012. http://www. mikekujawski.ca/2012/05/30/finally-some-2012-statistics-for-the-african-mobile-phone-market/ (accessed 20 November 2014)

23. Yeung J, Javidnia $\mathrm{H}$, Heley S, et al. The new age of play audiometry: Prospective validation testing of an iPad-based play audiometer. I Otolaryngol Head Neck Surg 2013;42:21. [http://dx.doi. org/10.1186/1916-0216-42-21]

24. Szudek J, Ostevik A, Dzieglielewski P, et al. Can uHear me now? Validation of an iPod-based hearing loss screening test. J Otolaryngol Head Neck Surg 2012;41(Suppl 1):S78-S84.

25. Khoza-Shangase K, Kassner L. Automated screening audiometry in the digital age: Exploring uHear . Khoza-Shangase K, Kassner L. Automated screening audiometry in the digital age: Exploring uHear
and its use in a resource stricken developing country. Int J Technol Assess Health Care 2013;29(1):4247. [http://dx.doi.org/10.1017/S0266462312000761]

26. Handzel O, Ben-Ari O, Damian D, et al. Smartphone-based hearing test as an aid in the initial evaluation of unilateral sudden sensorineural hearing loss. Audiol Neurootol 2013;18(4):201-207. [http://dx.doi.org/10.1159/000349913]

27. Berger EH, Killion MC. Comparison of the noise attenuation of three audiometric earphones, with additional data on masking near threshold. J Acoustic Soc Am 1989;86:1392-1403. [http://dx.doi. org $/ 10.1121 / 1.398699$

Accepted 10 November 2014. 